\title{
Is research painting a biased picture of implicit learning? The dangers of methodological purity in scientific debate
}

\author{
ROBERT C. MATHEWS \\ Louisiana State University, Baton Rouge, Louisiana
}

\begin{abstract}
The properties of implicit learning in natural settings are contrasted with those found in research. It is suggested that the search for pure cases and the necessity of finding features that clearly discriminate the two types of learning lead to bias in our estimation of the power of implicit processes. In more natural settings, such as face recognition, object perception, and natural language processing, implicit processes operate with flexibility and adapt to changes in environmental conditions. It is suggested that the search for pure cases of implicit processes has led to focusing on relevant but atypical examples of these processes. Additional research that emphasizes high levels of skill in control of complex systems may reveal greater adaptive power of implicit processes. However, such research may require less methodological purity and more emphasis on synthesis of theoretical ideas rather than analysis into pure cases.
\end{abstract}

Cognitive psychologists are better at analysis than at synthesis. We are experts at critical analysis, taking things apart. Analytical skills are the main substance of our experimental methodology and training. Similarly, scientific debates tend to focus our attention on narrower and (hopefully) clearer issues, as authors try to isolate phenomena and prove a point. Particularly in situations such as implicit learning, where there is an ongoing debate between believers and nonbelievers in the existence of a powerful cognitive unconscious, there is a tendency for both sides to seek especially clear or "pure" cases on which to make their stand. In the area of implicit learning, these tendencies have resulted in moving away from the central problem (e.g., nonconscious learning of natural language and other complex skills) to relevant but atypical situations and tasks (reaction time and categorization tasks). As a consequence, the picture being painted by current research of implicit learning may be biased in important ways.

Such possible biases have led research to suggest that knowledge associated with the cognitive unconscious is relatively "dumb." Research such as the Neal and Hesketh (1997) and Dienes and Berry (1997) papers in this issue suggests that implicitly acquired knowledge cannot be applied to new situations (e.g., transfer) without the helping hand of conscious control. One of the key features of implicitly acquired knowledge, according to current re-

Correspondence should be addressed to R. C. Mathews, Department of Psychology, Louisiana State University, Baton Rouge, LA 708035501 (e-mail: psmath@unix 1.sncc.lsu.edu). search, is that it is particularized. That is, it is tied to individual cases from prior experiences, and it does not generalize far beyond these cases. Dienes and Berry (1997) report evidence from experiments in dynamic system control suggesting that the implicitly acquired knowledge does not generalize at all beyond the exact cases experienced (e.g., levels of prior workers and resulting output). In addition, research suggests that implicitly acquired knowledge is fragmentary and incomplete. For example, the knowledge acquired about artificial grammars is often described as memories of pieces (bigrams and trigrams) of valid strings (Dulany, Carlson, \& Dewey, 1984; Perruchet \& Pacteau, 1990). Thus, compared with explicitly acquired knowledge, implicitly acquired knowledge is fragmentary, rigid, and weakly transfers. Finally, even when we have implicit knowledge, we do not know we have it. Consequently, subjects have no confidence in their implicitly guided decisions and think they are just guessing (Chan, 1992; Dienes \& Berry, 1997).

But consider implicit knowledge in everyday life, such as that involved in natural language processing. The language skills of most people are sufficiently well developed and complete enough to allow communication between strangers who happen to speak the same language. Language is fluid and flexible. People can generate an infinite number of sentences to communicate their ideas in new contexts. Of course, language is not pure implicit knowledge. However, all but a few school teachers (who insist on teaching the diagramming of sentences) strongly believe that language is primarily acquirer experientially rather than from explicit learning of language rules. As 
for confidence, most of us believe we are quite proficient in making ourselves understood. Perhaps, overconfidence rather than lack of confidence might better characterize natural, expert-level implicit knowledge.

Virtually all types of pattern recognition are acquired implicitly. Consider face recognition. Can you imagine consciously trying to figure out how you distinguish every person you know from every other person? Research on face recognition shows that it is a complex process involving feature extraction and development of structural descriptions at several different levels (e.g., see Burton \& Bruce, 1992). Some of the implicitly developed structural descriptions in face and object recognition, of which w'e are completely unaware, involve view-independent structural descriptions (Biederman \& Gerhardstein, 1993; Burton \& Bruce, 1992; Marr \& Nishihara, 1978; Perrett, Oram, Hietanen, \& Benson, 1994). These implicit perceptual processes operate flexibly and adaptively outside of conscious awareness and control.

Pattern recognition can also be highly adaptive and abstract. For example, searching for the letter $t$ in a background of $c \mathrm{~s}$ is much easier than searching for $t \mathrm{~s}$ in a background of several different letters (see Neisser, 1964). Yet the perceptual adjustment necessary to process minimal features of the letters in each context occurs automatically, without any conscious control. Also, it is not the case that this adaptive skill required to adjust processing of features was consciously controlled at an earlier point in perceptual development, before it became automatized. Conscious control over feature processing in perceptual search tasks is simply unnecessary. We automatically adjust to the search task demands, as demonstrated by the persistent evidence of implicit learning in novel perceptual search tasks (e.g., Cleeremans, 1993; Lewicki, Hill, \& Bizot, 1988; Stadler, 1989).

There have been many interesting experiments inspired by Gibson's (1977) ecological approach to perception that demonstrate both abstractness and transfer of implicit processes (e.g., see McCabe \& Balzano, 1986). For example, Johansson's (1973) experiments demonstrated that people have automatically abstracted invariant relationships between different body parts when viewing a person walking. He filmed a person with several light sources attached to different body joints. When the film was shown with the contrast turned all the way up the image appears to be a set of random dots. Yet, when the person being filmed begins to walk, the image is immediately perceived as a person walking. Even the gender of the person walking can be detected from the pattern of moving dots. Yet his subjects had never seen a person walking under such conditions prior to the experiment.

\section{Working Toward Synthesis}

In the remainder of this essay, I will attempt an exercise designed to help us get out of our usual critical analysis mode and work toward seeking synthesis. I highly recommend this exercise to readers as well. It is very simple. Just look for things you can agree with rather than focusing on oversights, flaws, and other obvious deficiencies in the papers being considered.

I agree with Neal and Hesketh (1997) that part of the problem may be the focus of attention in implicit learning research on the issue of consciousness. Consistent with this effort to avoid focusing on the consciousness issue, I prefer to use the term experiential knowledge, avoiding the implication that any implicitly acquired knowledge must be completely nonconscious. I also agree with Dienes and Berry (1997) that there is strong evidence that people can have implicitly acquired knowledge at the subjective criterion of being nonconscious. However, finding clear cases where implicitly acquired knowledge is completely nonconscious by a subjective threshold does not imply that implicitly acquired knowledge is always nonconscious or that we can have no explicit access to it. Most theorists of implicit learning tend to overlook the consistent finding that some implicitly acquired knowledge can be verbalized, and the amount verbalizable tends to increase as a function of practice (e.g., Mathews et al., 1989; Stanley, Mathews, Buss, \& Kotler-Cope, 1989). Rather than assume that knowledge is acquired simultaneously and independently by implicit and explicit learning processes, it makes more sense to me to believe that the two systems interact. For example, by consciously reflecting about how we are performing a task, we often can discover (make explicit) our own implicit knowledge. Just because information was acquired implicitly does not mean it can never become explicit.

I think the problem is bigger than refocusing on intention rather than consciousness, as suggested by Neal and Hesketh. The concept of intentional control is likely to be every bit as complex and slippery as the concept of consciousness. For example, consider the difficulty lawyers have in criminal or civil cases attempting to prove intention. Intentional control is likely to depend on attentional control. We already have considerable evidence suggesting that there is no single mechanism of attentional control (e.g., Allport, 1993; Posner \& Petersen, 1990).

The problem is really in focusing per se. That is, the problem lies in seeking a critical feature and pure cases where implicit and explicit knowledge exist as completely separate entities. Our research methods bias us to look for "it," the one true feature that will clearly separate implicit from explicit learning. As students of human knowledge, we should know better. We have already learned from the research on natural categories that there are often no critical discriminating features that can be used to distinguish between categories (e.g., Fodor, Garrett, Walker, \& Parkes, 1980; Rosch \& Mervis, 1975; Wittgenstein, 1958). For example, there is no one feature that can be used to distinguish all cups from all bowls; yet we still find the concept useful.

The debate between believers and nonbelievers in implicit learning is similar to the debate between a defense lawyer who claims "evidence is like a chain" versus the prosecution who claims "evidence is like a rope." Nonbelievers argue that there is no one, key, indisputable piece 
of evidence for implicit learning. Believers, like Dienes and Berry, suggest looking at the extensive patterns of evidence and argue that there is extensive support for the distinction. Since I am more interested in what we can do with the concept of two types of learning (its affordances) than whether it actually exists in neural tissue, I side with Dienes and Berry. Whether the concept is made of ropes or chains, I think it has valuable applications for understanding failures in education and communication (see Norman, 1993).

The evidence cited by Dienes and Berry (1997) that implicitly acquired knowledge lacks confidence is very interesting. It seems to point to a relatively pure case for differentiating the two types of knowledge. However, as mentioned above, I doubt that lack of confidence is always associated with implicit knowledge. Sometimes we are quite sure that we know what we are doing even though we cannot explain why we do it that way. Perhaps, lack of confidence is characteristic of early stages of implicit knowledge acquisition. This is another one of those points where we must be careful not to characterize all implicit knowledge in terms of a quality that is useful for discriminating clear cases of the two types of learning.

\section{An Evolutionary Perspective on the Qualities of Implicitly Acquired Knowledge}

I believe that the most important functional divide in types of human knowledge occurs at a much higher level than that associated with contemporary models of implicit learning. On the basis of evidence of human evolution (e.g., Corballis, 1991; Donald, 1991), it appears that intentional mental control evolved before development of our verbal/symbolic system. Even though knowledge is acquired implicitly at the mimetic level, as explained below, it is, to some degree, conscious and under some level of intentional control when being applied.

Donald (1991) describes several major developments in terms of evolving human knowledge systems. Our earliest ancestors had abilities to abstract functional cues from the environment for their survival. These earliest capabilities for procedural learning are still shared with all animal species today (e.g., conditioning). Next for man came episodic knowledge, the ability to preserve and remember specific past experiences as a guide for future behavior. Early episodic knowledge was highly particularized (not abstract) and stimulus dependent. Only when appropriate cues were available to remind one of a past experience could episodic knor ledge be remembered and used in a new situation. However, that decisions would be necessary in determining how to react or apply past experiences to new situations suggests that some level of conscious awareness evolved at this stage.

Creative or self-generated control of thinking begins, according to Donald, at the mimetic stage, still a long time before symbolic thought and language evolved. At this point, early humans began to mime or reenact important events. This miming of events provided an opportunity to re-present events, and successive enactments could be altered across time. The adaptive value of mime lies in its ability to rehearse and, perhaps, improve upon behaviors. It also opens the door for increased communication. While mimetic knowledge begins with reenactments of specific events (episodic knowledge), this form of knowing became specialized for communication and preservation of culture rather than accurate preservation of personal past events. Since conscious control of knowledge acquisition would seem to require symbolic thought, these forms of learning are implicit rather than explicit. However, the acquired knowledge could be explicit in the sense that people were aware of having memories or miming behaviors. Also, they had some intentional control over how this knowledge was applied.

This synthesis suggests that several different types of implicitly acquired knowledge might exist, each with different sets of properties (see Mathews \& Roussel, in press). However, I believe that the most important functional distinction for understanding human thinking lies between all of the earlier forms of knowing (procedural, episodic, and mimetic) versus verbal symbolic thought. Following Norman (1993), we refer to the former as experiential knowledge and the later as reflective knowledge. The reason this distinction is important is because it is related to natural dissociations that cause major difficulties for learning and communication among people. The problem arises from the different qualities and special functions of the two learning types. Experiential learning is especially adapted for action (i.e., doing the right thing at the right time). Reflective knowledge is especially adapted for preserving and transmitting knowledge to different people and across generations. Thus, experiential knowledge tends to be tied directly to environmental cues that guide behavior: It is highly contextualized. Reflective knowledge is less contextualized (e.g., tied to cues in the environment) and depends on more slippery verbal concepts and symbols that are open to reinterpretation. Thus, in everyday life, we often have problems teaching in ways that the knowledge will be used effectively (using reflective knowledge as a guide for doing) and figuring out how to conceptualize and communicate important ways of doing (making experiential knowledge explicit or reflective). The relative independence in learning of these two types of knowledge sometimes makes it very difficult to impact performance by verbal instructions (e.g., Mathews et al., 1996; Stanley et al., 1989).

The most powerful form of experiential knowledge (mimetic knowledge) has the following properties. (1) It consists of recombinable fragments. (2) It can transfer to new situations when the same invariant relationship applies. (3) The combinations of fragments used to construct wholes are internally constrained. That is, the constraints imposed on combination of pieces are not determined by nature ("I never saw a thing like that") or limits of the imagination ("I cannot imagine such a thing"). Instead, it is constrained by the implicitly acquired, informal rules of the culture in which the knowledge is embedded. 
(4) Finally, such knowledge is generative. It is capable of producing new and appropriate (not random) combinations that are different from any experienced before.

\section{The Challenge}

I would like to pose the following challenge for researchers interested in furthering our knowledge of implicit learning. If, as I have argued, natural implicit learning is not necessarily as particularized, rigid, and limited as the current research suggests, how can we design experiments to expose the extent of these qualities? How can we demonstrate that implicit learning can be fluid and adaptable?

I have a few hints to offer. First, natural language development also demonstrates times of overrigidity in application of rules-particularly when they are first acquired. It could be that implicitly acquired rules are rigidly applied at first, and flexibility comes later with additional practice. Alternatively, it could be that rigidity comes when rules are first crystallized as explicit concepts. Thus, it could be explicit, rather than implicit, learning that was responsible for rigidity. We badly need experiments that will examine qualities of implicitly acquired knowledge at higher levels of practice and skill development than typically found in the classic $1-\mathrm{h}$ experiment.

Second, experiments on implicit learning should involve complex mental thought and actions. Rather than simply picking strings as good or bad and grammatical or not (as in typical passive artificial grammar experiments), subjects should generate valid strings or control dynamic systems. The more complex the concepts involved and the more active skills employed, the more we are likely to find the true capabilities of implicit learning. The evidence cited by Dienes and Berry concerning finding less implicit learning when subjects simply watch someone perform a task or have someone else punch in their answers is highly relevant here. You cannot test a race car on a city street; we need to study complex behaviors on complex tasks.

Transfer depends on the conditions of training. Like picking out $t \mathrm{~s}$ in different backgrounds, implicit systems adapt to task demands and opportunities. Experiments that can demonstrate the power of implicit abstraction require tasks that demand it and experience (e.g., variability of practice situations) that can produce it.

Finally, we need to encourage studies and methods of synthesis rather than just analysis. Modeling offers one opportunity to test combinations of ideas about learning. However, we need to bring a synthesis mode of thinking into play in exploring potential models rather than our typical critical mode of thought (e.g., "this model is wrong because ..." or "my model is better than yours because ..."). For example, classifier systems provide a useful architecture for combining ideas about implicit and explicit learning (see Mathews \& Roussel, in press). Classifiers are simply sets of "if-then" rules that compete for controlling behavior of a system. Within classifier systems, knowledge can be both particularized and abstract (contain very specific rules and more general rules). Rules acquired from examples using the forgetting algorithm (Mathews, 1991) are both fragmentary and highly contextualized. Consciousness can be simulated as a small set of rules (e.g., the highest bidding set of 10 rules) that have their conditions matched at any point in time. Implicit or nonconscious rules can be modeled as rules whose actions become conscious, while the conditions that triggered them remain unconscious. The virtues of classifier systems are that we can add together known qualities of implicit and explicit knowledge systems, test the effects of different changes to a system, and exchange knowledge between human and machine (the model) relatively easily. That is, such modeling architectures provide a tool for synthesis of ideas about implicit systems.

Modeling is only one way of seeking synthesis. Others include attempting to keep in contact with archetypal examples of what it is you are attempting to study (e.g., natural language learning in the case of implicit learning). Using theoretical perspectives outside a domain, like applying evolution theories to implicit learning (Reber, 1993), can also be very valuable. I believe that progress in understanding implicit learning will require using such synthesis tools, combined with our already highly developed skills in analysis.

\section{REFERENCES}

Allport, D. A. (1993). Attention and control: Have we been asking the wrong questions? A critical review of twenty-five years. In D. E. Meyer \& S. M. Kornblum (Eds.), Attention and performance XIV (pp. 183-218). Cambridge, MA: MIT Press.

Biederman, I., \& Gerhardstein, P. C. (1993). Recognizing depthrotated objects: Evidence for 3-D viewpoint invariance. Journal of Experimental Psychology: Human Perception \& Performance, 19, $1162-1182$

Burton, A. M., \& Bruce, V. (1992). I recognize your face but I can't remember your name: A simple explanation? British Journal of Psychology, 83, 45-60.

Chan, C. (1992). Implicit cognitive processes: Theoretical issues and applications in computer systems design. Unpublished doctoral dissertation, University of Oxford.

Cleeremans, A. (1993). Mechanisms of implicit learning: Connectionist models of sequence processing. Cambridge, MA: MIT Press.

Corballis, M. C. (1991). The lopsided ape. New York: Oxford University Press.

Dienes, Z., \& Berry, D. (1997). Implicit learning: Below the subjective threshold. Psychonomic Bulletin \& Review, 4, 3-23.

DonALD, M. (1991). Origins of the modern mind: Three stages in the evolution of culture and cognition. Cambridge, MA: Harvard University Press.

Dulany, D. E., Carlson, R. A., \& Dewey, G. I. (1984). A case of syntactical learning and judgment: How conscious and how abstract? Journal of Experimental Psychology: General, 113, 541-555.

Fodor, J. A., Garrett, M. F., Walker, E. C. T., \& Parkes, C. H. (1980). Against definitions. Cognition, 8, 263-367.

Gibson, J. J. (1977). The theory of affordances. In R. E. Shaw \& J. Bransford (Eds.), Perceiving, acting, and knowing (pp. 67-82). Hillsdale, $\mathrm{NJ}$ : Erlbaum.

JOHANSSON, G. (1973). Visual perception of biological motion and a model for its analysis. Perception \& Psychophysics, 14, 201-211.

Lewicki, P., HiLl, T., \& Bizot, E. (1988). Acquisition of procedural knowledge about a pattern of stimuli that cannot be articulated. Cognitive Psychologv, 20, 24-37. 
MARR, D., \& Nishihara, K. (1978). Representation and recognition of the spatial organization of three-dimensional shapes. Philosophical Transactions of the Royal Society of London: Series B, 200, 269-294.

MATHEws, R. C. (1991). The forgetting algorithm: How fragmentary knowledge of exemplars can abstract knowledge. Journal of Experimental Psychology: General, 120, 117-189.

Mathews, R. C., Buss, R. R., Stanley, W. B., Blanchard-Fields, F., Cho, J. R., \& Druhan, B. (1989). The role of implicit and explicit processes in learning from examples: A synergistic effect. Journal of Experimental Psychology: Learning, Memory, \& Cognition, 15, 1083-1100.

Mathews, R. C., Lane, I. M., Roussel, L. G., Nagy, M. S., HaptonSTAHL, D. E., \& BROCK, D. B. (1996). Using conscious reflection, group processes, and AI to facilitate development of expertise. Journal of Experimental \& Theoretical Arificial Intelligence, 8, 259-276.

Mathews, R. C., \& Roussel, L. G. (in press). Abstractness of implicit knowledge: A cognitive evolutionary perspective. In D. Berry (Ed.), How implicit is implicit learning? New York: Oxford University Press.

McCabe, V., \& Balzano, G. J. (EDS.) (1986). Event cognition: An ecological approach. Hillsdale, $\mathrm{NJ}$ : Erlbaum.

Neal, A., \& Hesketh, B. (1997). Episodic knowledge and implicit learning. Psychonomic Bulletin \& Review, 4, 24-37.

NeISSER, U. (1964). Visual search. Scientific American, 210, 94-102.

Norman, D. A. (1993). Things that make us smart: Defending human attributes in the age of the machine. Reading, MA: Addison-Wesley.
Perrett, D. I., Oram, M. W., Hietanen, J. K., \& Benson, P. J. (1994). Issues of representation in object vision. In M. J. Farah \& G. Ratcliff (Eds.). The neuropsychology of higher vision: Collected tutorial essays (pp. 33-62). Hillsdale, NJ: Erlbaum.

Perruchet, P., \& Pacteau, C. (1990). Synthetic grammar learning: Implicit rule abstraction or explicit fragmentary knowledge? Journal of Experimental Psychology: General, 119, 264-275.

Posner, M. I., \& Petersen, S. E. (1990). The attention system of the human brain. Annual Review of Neuroscience, 13, 25-42.

ReBER, A. S. (1993). Implicit learning and tacit knowledge: An essay on the cognitive unconscious. New York: Oxford University Press.

Rosch, E., \& Mervis, C. B. (1975). Family resemblances: Studies in the internal structure of categories. Cognitive Psychology, 7, 573-605.

Stadler, M. A. (1989). On learning complex procedural knowledge. Journal of Experimental Psychology: Learning, Memory, \& Cognition, 15, 1061-1069.

Stanley, W. B., Mathews, R., Buss, R., \& Kotler-Cope, S. (1989). Insight without awareness: On the interaction of verbalization, instruction and practice on a simulated process control task. Quarterly Journal of Experimental Psychology, 41A, 553-577.

Wittgenstein, L. (1958). Philosophical investigations (2nd ed.). Oxford: Blackwell.

(Manuscript received August 20, 1996; revision accepted for publication October $31,1996$. 